\title{
Parametric Connotation and Theoretical Extension of Parametric Architecture Aesthetics
}

\author{
Liangwa Cai \\ School of Architecture, Tianjin University, Tianjin, China
}

\section{Email address:}

cailw@163.com

\section{To cite this article:}

Liangwa Cai. Parametric Connotation and Theoretical Extension of Parametric Architecture Aesthetics. Science Innovation. Vol. 5, No. 1, 2017, pp. 15-20. doi: 10.11648/j.si.20170501.13

Received: January 4, 2017; Accepted: February 21, 2017; Published: March 3, 2017

\begin{abstract}
Parametric Design has aroused the innovation in the architectural form creation, space conception and technology conception as a sort of new thinking mode. Coming along with this situation, architectural aesthetics has already started a new step in the influence of parametric design. Using the basic principles of philosophy, aesthetics, information technology, from the Multidimensional Vision systems theory, information theory, behaviour psychology, philosophical thinking and logical induction dual perspective of parametric architectural beauty philosophical connotation, aesthetic principles, aesthetic value and artistic regularity analysis, so as to construct a theoretical framework parameterized Aesthetics. Proposed integrity and process, interaction with equality and pluralism and individuality is an important aesthetic value principle parametric architectural aesthetics. And from complex parametric building phenomenon, summed parametric architectural aesthetics content evolution include: aesthetic dimension in the form of expansion of the connotation of spatial variation in the type of aesthetic and technical aesthetics extend and so on. Expanding the creative vision of today's architectural and aesthetic theory could provide new ways of thinking, and provide a theoretical basis for aesthetic parametric building program evaluation.
\end{abstract}

Keywords: Parametric Architecture Aesthetics, Philosophical Connotation, Aesthetics Principle, Extension of Conception

\section{参数化建筑美学的哲学内涵与理论拓展}

蔡良娃

建筑学院, 天津大学, 天津, 中国

邮箱

cailw@163. com

摘要: 参数化设计代表了一种全新的思维模式, 为建筑的形体生成、空间观念和技术观都带来了不同以往的变革, 随 之而来, 参数化思潮影响下建筑艺术的审美也进入了全新的阶段。运用哲学、美学、信息技术等基本原理, 从系统论、 信息论、行为心理学的多维视野, 以哲学思考和逻辑归纳双重角度, 对参数化建筑美的哲学内涵、审美原则、审美价 值以及艺术规律进行了分析, 从而建构参数化美学的理论框架。总结了参数化建筑美学哲学内涵; 并从纷繁复杂的参 数化建筑现象中, 总结出参数化建筑在形式美学、审美意象与审美评价等方面的演变。为拓展当今建筑创作视野, 提 供全新的美学理论和思维方法, 并能为参数化建筑方案评价提供美学理论依据。 


\section{1. 引言}

参数化技术是应用数字技术, 通过确立个因子之间的 相互关系, 进行可调整、可控制的动态操作辅助设计平台。 当前, 建筑界对数字化手段的应用, 从最早的计算机辅助 设计演变到基于算法的参数化设计阶段。参数化设计思潮 指引着新锐设计师, 从旧有的建筑设计模式中解脱出来, 运用更加灵活、更加富有想象力、更加精确的方法进行设 计。建筑的形态与空间开始向着更流动、更模糊、更复杂 的方向发展。同时, 随之改变的不仅仅是建筑的面貌, 也 包括了建造、设计方法、设计理论等的各种内容。正如尼 尔・林奇 (Neil Leach) 在《非物质化过程》 (IMMATERIAL PROCESSES) 一文中指出, 世纪之交, 越来越多的建筑师和 工程师开始使用计算机的 “非物质” 逻辑去解决实际建造 的 “物质” 问题。[1]数字技术和建构旧的对立已经开始 崩溃, 数字技术正在越来越多地用于为建构服务。

参数化建筑设计的重心从设计成果转移到设计规则, 从关心设计的结果到关心设计的过程。参数化设计工具与 方法本质上的改变, 影响建筑设计的思维方式与审美趋向, 建筑的审美价值观、审美时空观和创作观等都发生着不同 以往的变革。[2]从纷繁复杂的参数化建筑现象中, 总结 参数化建筑美学的哲学内涵与美学特征, 能为拓展当今建 筑创作视野, 提供全新的美学理论和思维方法, 并能为参 数化建筑方案评价提供美学依据。

我们认为参数化建筑美学是数字技术影响下正在逐 渐形成的美学理论, 从研究内容来看, 参数化建筑美学以 信息论、系统论、控制论、行为心理学等科学理论为哲学 依据探索建筑与环境的美学规律, 分析建筑艺术与社会、 经济、文化的审美关系, 从而把握建筑要素的审美信息的
产生和构成特征。[3] 它关注建筑及其环境的审美信息的 交流与反馈的规律, 致力于研究数字化技术影响下建筑和 城市空间观念、空间结构所发生的种种变化, 探索社会审 美文化和人们的审美意识的演进与发展。

\section{2. 参数化建筑美学的哲学内涵}

参数化建筑的审美活动中, 审美主体与客体的关系发 生了根本性的变化, 不再是对立的主客关系, 而是主体与 主体的同一关系, 也就是 “主体间性”。参数化建筑设计 与媒体学、生物学、神经学等紧密联系, 光、水、风、运 动等无固定形态的形式内容和声音传播、荷尔蒙分泌、神 经传递等非形式性的内容越来越多地参与到建筑空间的 构建中 $[4]$ 。

作为一种建筑方案初期的环境性能模拟平台, Ecotect提供了一种交互式的分析方法, 只要输入简单的 模型, 就能提供建筑室内外物理环境的数字化的可视分析 图。在参数化建筑设计领域中, 建筑光环境 (主要为日照 及遮阳）涉及到地域环境的舒适度问题, 是目前研究较为 广泛深入的一个领域。借助环境性能模拟技术 (如Ecotect、 Fluent、CFD等软件) 来实现建筑主动适应与调节气候的 目的。通过对当地日照、气流、辐射、声场等环境的模拟， 得出建筑周围的气流流场、日照、温度等变化, 将此参数 提取输入设计软件来选择合适的建筑布局、细部构造形式、 控制形体生成、表皮变化。在BIG事物所设计的阿斯塔纳 国家图书馆（Astana National Library）项目中, 就应 用了Ecotect对太阳辐射进行分析, 并以此参数作为建筑 表皮开窗依据（图1）。
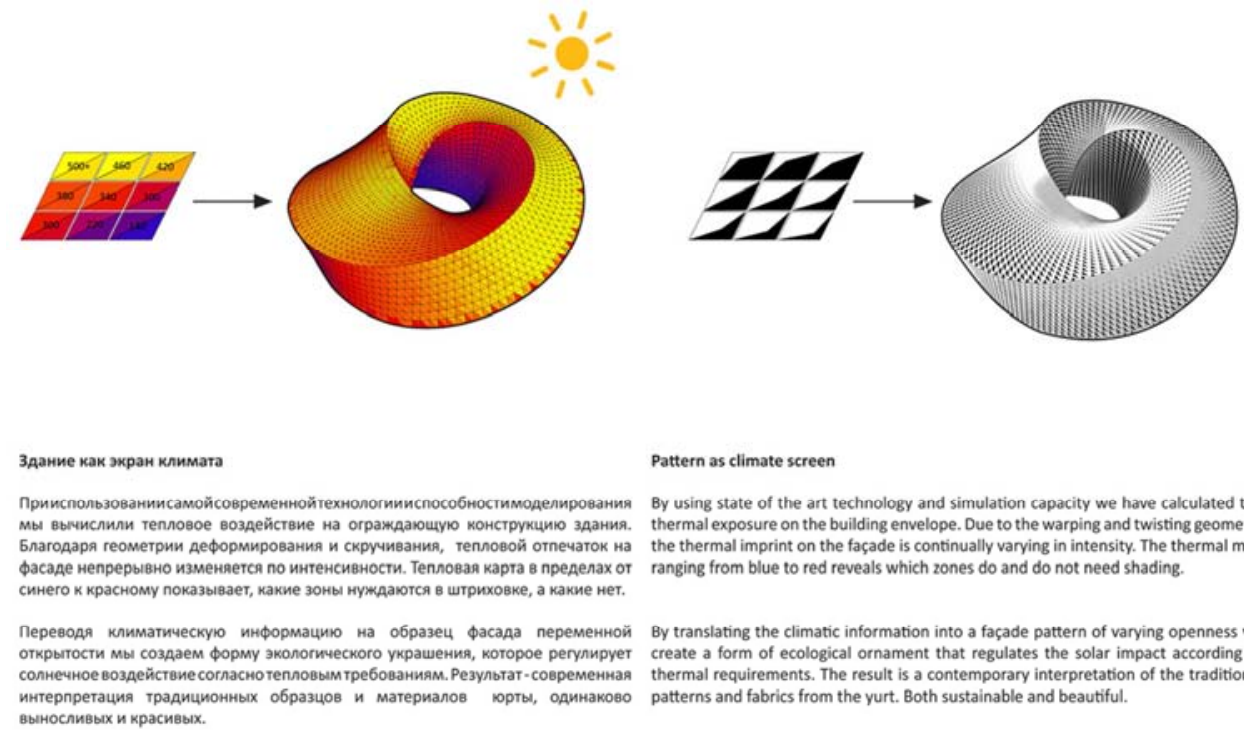

By using state of the art technology and simulation capacity we have calculated the thermal exposure on the building envelope. Due to the warping and twisting geometry the thermal imprint on the façade is continually varying in intensity. The thermal map ranging from blue to red reveals which zones do and do not need shading.

By translating the climatic information into a façade pattern of varying openness we create a form of ecological ornament that regulates the solar impact according to thermal requirements. The result is a contemporary interpretation of
patterns and fabrics from the yurt. Both sustainable and beautiful.

图1阿斯塔纳国家图书馆日照分析图。

参数化建筑设计将人的动态活动与所需空间联系在 一起, 通过对以往各学科知识的综合处理, 得到建筑形体。 建筑形体与人的动态活动相关联, 一旦人的活动流线、形 式、组合方式等发生了改变, 建筑形体也会随之发生变化。
NOX的作品SON-0-House是一个可以举行集会的音乐构件 装置（图2）, 在这个音乐艺术品装置中, 当其中一个 声音发生之后, 会被分布设置在建筑中的各个传感器捕 捉到, 并进行处理。在传感器作用下, 声音及在屋内活 
动的人声被捕捉到, 从而又创造出新的声音效果。来访 者的运动不断地被遍布建筑中的传感器探知, 其测算结果 被储存在一个持续更新的数据库, 已经合成的声音就能在 新组合中得以重编, 即所谓互动。这样一来作曲就成为一 个进化的记忆景观，随着参观者在建筑空间中的行走行为 而持续发展。

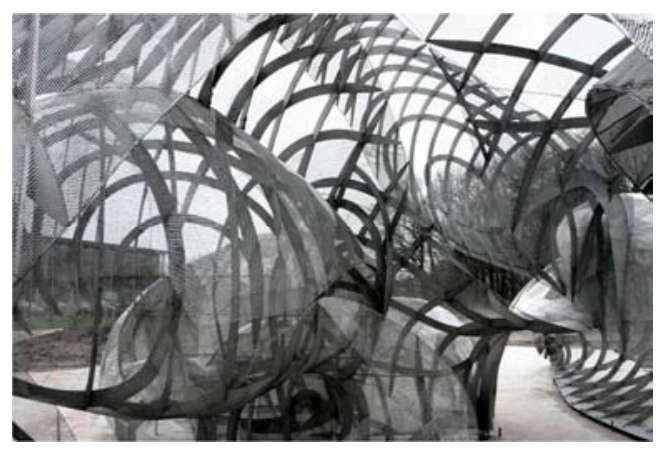

图2 Son-0-House局部。

参数化设计强调的是建立联系各参量的逻辑规则, 注 重主体与主体之间的理解关系; 不是注重普遍规律, 而是 注重特殊情况即个性; 不是外在的客观认知, 而是对意义 的体验和理解。这实际上把参数化设计活动当作是主体与 主体间的理解活动, 具有主体间性, 即自我与世界作为互 相交往的主体，通过精神层面的交流对话，达到审美的同 生共在, 最终形成一元主体, 具有主体间性 [5]。参数化 建筑作品更加注重体验性、交流性、参与性, 这种积极的 交流和互动使得建筑作品意义的阐释变得多元而丰富, 使 建筑的体验与解读过程充满审美的愉悦。

建立在主体性上的传统建筑审美活动是主体 (建筑欣 赏、使用者）对客体对象 (即建筑) 的认识、情感投射或征 服, 把建筑师所反映的精神现象作为对立的客体世界，与 主体形成二元对立关系。而建立在主体间性上的参数化建 筑审美活动中, 建筑艺术形象不再是与我无关的客体, 自 我主体和对象主体共同合为一体, 形成一元关系。当我们 在从事 (或欣赏) 建筑审美活动时, 一开始把建筑当作客体, 就如在现实生活中把他人或世界当作客体一样, 以一种外 在的立场看建筑对象, 但随着建筑审美体验的深入, 会逐 渐进入审美的自由境界。审美理解、审美同情等因素使自 我消失, 自我意识变成对象意识, 建筑艺术形象的客体性 也消失, 成为另一个主体, 并通过与自我的交流进入自我 之中, 对象意识成为自我意识。这时, 主体不仅在看建筑 艺术形象, 而是与建筑艺术形象共同成为审美活动的主体, 实现了建筑审美的主体间性。建立在主体间性理论基础上 的参数化建筑的审美意义, 更强调存在的本质一一主体与 主体间的关系, 重视自我主体与对象主体间的直觉体验和 对话交流。

\section{3. 参数化建筑的美学特征}

\section{1. 参数化建筑中形式美学的秩序性}

秩序性是美学范畴的一个关键问题, 秩序是最易感 悟和最具审美价值的审美对象。审美心理学领域的研究
表明, 审美快感来自于对某种介于乏味和混乱之间的形 式的欣赏。西方传统审美观念认为, 秩序程度越高, 则 对象越美。

现代主义建筑遵从欧几里德的几何美学, 认为简明、 单一、线性的秩序具有审美价值, 认为基本几何形体, 如 立方体、圆柱体、圆锥体、棱雉体、球形体、正三角形体 等 “完形” 是最具美学价值的形式。（图3） “模度” 是
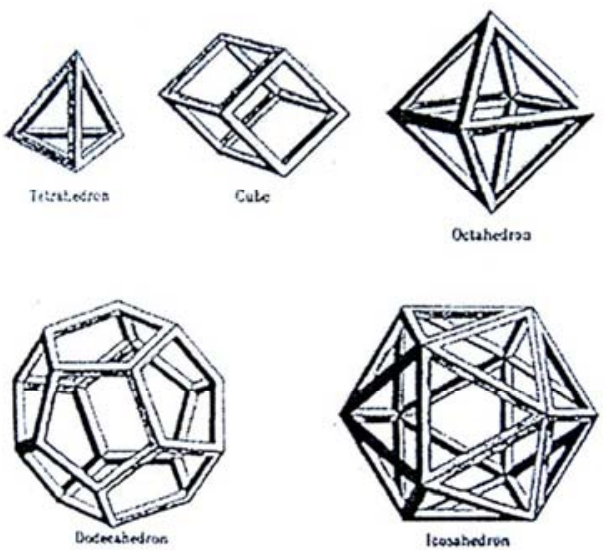

图3 柏拉图本体论的永恒空间 “完型” 形式。

柯布西耶的秩序观，他采用了 “模度” 来控制其设计工作 并带来秩序。（图4）他认为, “模度” 的运用, 是许多 设计工作变得异常的简单, 设计中的许多犹豫和不确定, 甚至错误都被提前解决。在《走向新建筑》中, 柯布这样 说过: “一个模数赋予我们衡量与统一的能力, 一条控制 线使我们能进行构图而得到满足。” 后现在建筑追求的是 复杂、多义、多元的秩序美学。数学家伯克霍夫就在《美

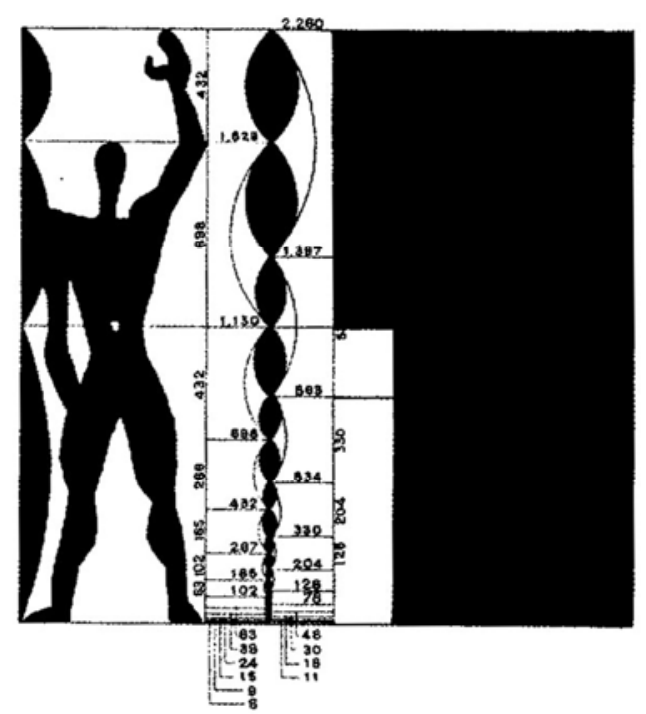

图4 柯布西埃模度图示。

学标准》中提出的衡量美的量化方式——审美度”: 美学 价值M与其秩序感 0 (Order) 成正比, 与其复杂程度 C (Complexity) 成反比, 即 $M=0 / C$ 。也就是说, 秩序与复 杂是相伴相生的, 秩序需要复杂性才得以彰显, 秩序与复 
杂性一种互补关系 [6]。与现代建筑中功能决定形式的简 明秩序，以及后现代主义追求含混、多元与折衷的复杂秩 序不同，参数化设计追求的是复杂系统中的潜在、隐含秩 序。[7]在《建筑复杂性和矛盾性》一书中, 文丘里阐述了 后现代建筑对含混与折衰的变化秩序的追求: 我喜欢建筑 要素的混杂, 而不要“纯净’; 宁愿一锅煮, 而不要清爽的; 宁要歪扭变形的, 而不要直截了当的; 宁要暧昧不定, 而 不要条理分明、刚愎、无人性枯燥和所谓的有趣; 我宁愿 要时代相传的东西, 也不要经过设计的; 要随和包容, 不 要排他性......否认建筑艺术中的既有简单秩序, 排斥逻辑 性, 宣扬复杂性, 以杂乱、怪诞和暧昧为美是后现代主建 筑美学的一大特色。（图5）文丘里设计的福林特住宅就 把古典柱式变成了轮廓剪影式的平面形状, 鼓胀、矮胖的 形象令人忍俊不禁。（图5）

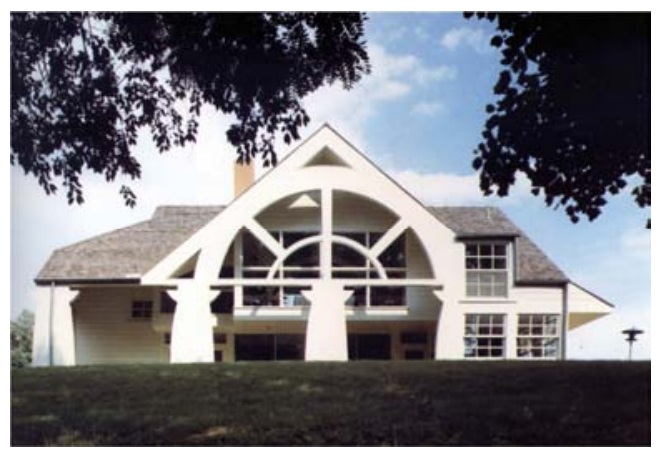

图5 福林特住宅立面设计。

当代参数化建筑深受复杂科学影响, 其科学范式促使 人们从关注系统中的各孤立要素, 转变为研究各要素之间 的关系。复杂性科学强调建立学科之间的横向联系, 使当 代建筑师认识到仅靠传统的设计方法已不足以应对大量 随机的、复杂的设计问题 [8], 建筑学与其他学科的融合 与交叉成为必然的选择。清华大学建筑设计研究所所长徐 卫国对参数化建筑设计做出的解释为: 所谓计算机参数化 设计实际上就是要找到一种关系或称规则, 用这一关系或 规则来模拟影响建筑设计的某些主要因素表现出的行为 或现象。参数化建筑设计试图寻找复杂系统中的隐含秩序, 诸如: 参数化建筑与其所处环境之间的相互联系; 与其所 在地段之间的相互联系; 在其功能系统、结构系统和设备 系统设计中所要考虑的相互联系; 参数化建筑在形式系统 设计中所要考虑的各组成部分之间的相互联系等。参数化 设计跳出了现代主义设计方法的框架, 转向对无序的、非 平衡态和不可逆的设计要素作严格的逻辑分析。参数化设 计中, 设计师在分析和研究设计条件时, 就是在寻求逻辑 关系的过程, 在寻求和调整设计条件的逻辑关系下得到设 计结果, 这种抽象的逻辑关系输入条件后输出形态, 会给 人们带来始料末及的建筑形体效果。

自然界中的生物、植物都呈现出特定的形态，在这些 千变万化的形态背后, 却有极其严密而规整的自身秩序进 行控制。当代建筑师在研究其内部逻辑的基础上, 通过参 数化算法等数字技术用于建筑生成。将自然界中的隐含的 美学秩序转译为控制建筑形体生成的参数, 形成了参数化 建筑设计中的形态逻辑。约翰 - 福拉则 (John Frazer) 将
遗传算法 (Genetic Algorithm) 引入建筑学领域, 提出了 基于非线性科学思想的进化建筑学 (Evolutionary Architecture), 它将自然界的进化模型用于建筑形式的 生成, 以基因规则表示的建筑被看成是一种人工生命体的 形式。他在1995年伦敦建筑学会发表的一篇文章《生成和 进化设计的数字代码脚本》中, 展示了 “网络进化设计系 统（Networked Evolutionary Design System）” [9]（图 6）遗传算法的关键就是把设计目标的生成过程分解为一 定的逻辑过程或步骤, 并发现其内在的基本秩序一一遗传 代码, 这些秩序也是设计的 “遗传因子”。根据遗传算法 生成的建筑, 形式秩序的产生是由建筑生成的内在组织发 展逻辑过程组成。

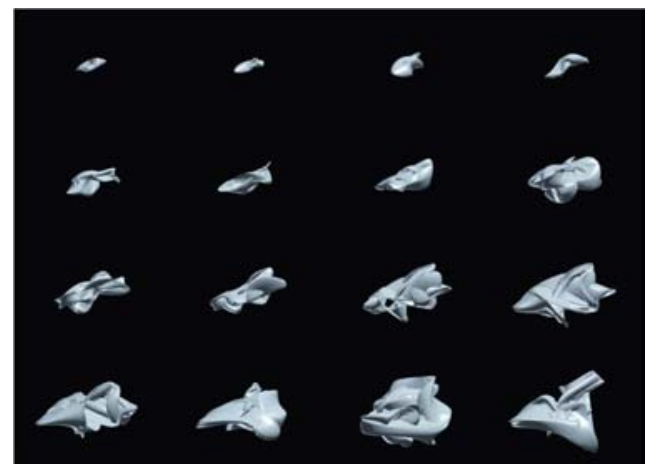

图6网络进化设计系统

\section{2. 参数化建筑美学的审美意象}

意象可分解为 “意” 和 “象”二部分： “象” 指形象, 是可见的物质内容; “意” 是意义、意思、情意, 指思想 感情, 是不可见的精神内容。建筑审美意象是借助于建筑 的具体形象来言情表意, 无论是以建筑的实体和空间体现 出来的艺术形象, 还是人脑中的映象, 都离不开具体的物 质载体, 一切建筑意象都具有具体建筑形象, 也就是说 “意” 涵在 “象”内。建筑师常利用形状的象征性引起观众联想, 小沙里宁设计的纽约环球航空公司候机楼像一支展翅欲 飞的大鸟, 悉尼歌剧院则像一艘迎风而驰的帆船、海滩上 的贝壳。而人们在感受建筑时存在着观赏者自身情趣和联 想的多样性以及品味的高低。建筑意象具有明显的模糊性、 多义性, 在一定范围里总是 “一象多意” , 如柯布西耶的 朗香教堂就是一个主题朦胧, 多义的作品, 多种可能的作 品。 [10]修肯 $(H \cdot$ Schocken) 在AA建筑学院的一次课堂 讨论中, 让学生们对朗香教堂的造型发表评论（图7）, 学 生们对这座教堂造型看法不一, 产生了 “鸭子”、“白帆 船”、“轮船”、“祈祷的双手”、“教士的帽子”、“修 女的头巾”、“蟹壳”、“信徒的耳朵” 等联想。 [11]

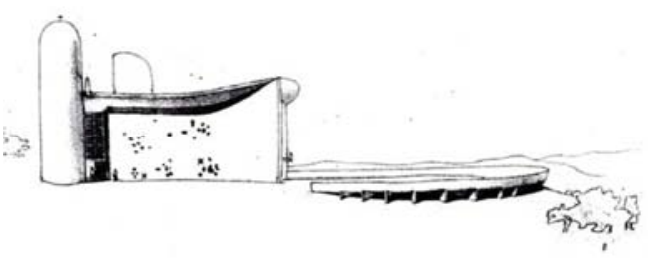

图7 朗香教堂草图。 
而科学创造中每一新公式、模型、定理等作品的诞生, 都是以科学独有的表达式所显示的科学意象的诞生。它源 于科学家所直观到的客观意象, 同时又因其在内部对 “意” 的高度抽象概括性, 其 “象” 和结果的符号化, 使之比艺术 作品以艺术形式所显示的审美意象, 更远离了最初的直观 意象。以物理学中霓虹现象到反射原理的发现为例就有: 在科学观察阶段面对客观的 “眼中霓虹” ，在头脑中经科 学思维加工成科学数据和图像的 “心中霓虹” , 抽象成反 射原理及数学模型, 将霓虹及类似现象的共同反射原理, 表达成定理或公式的 “手中霓虹”。[12]

参数化建筑设计是一个科学研究和解决问题的过程, 它与科学意象的生成过程更加类似, 始终以 “意” 为主体 展开, 力图将象中之 “意” 抽取和概括起来, 发现其本质 规律或建构数学模型, 当改变变量时, 就出现了 “多象一 意” 的结果。数字技术的迅速发展, 使数学概念、逻辑、 公式等, 在数字环境下转换成具体形态, 成为一种崭新的 造型方法, 在无形与有形之间建立起了直观的联系。

建筑师不是直接控制形态的变化, 而是通过数学公式 或参数的改变控制生成逻辑的变化, 从而间接地控制形态 的生成。面对建筑设计的复杂性时, 与建筑密切相关的所 有设计因素都有可能在特定的问题中成为重要参数。设计 师分析建筑建筑功能、形式、结构、环境行为心理等客观 条件, 探究其内部逻辑规律, 定义关键参数与逻辑关联, 转换为程序、算法、公式等生成逻辑, 也就是高度抽象概 括出参数化建筑 “意”。当建筑师操作设计中的生成规则 与所设置的参数变量时, 生成的结果也就是建筑形体的 “象” 是无穷多解而不是单一解。

其中最有意义的一项尝试就是, 建筑师试图将传统地 域建筑中的隐含的美学秩序转译为控制建筑形体生成的 参数, 生成了参数化地域建筑设计中的形态逻辑。在寻求 和调整设计条件的逻辑关系下得到设计结果, 这种抽象的 逻辑关系输入条件后输出形态, 会给人们带来始料未及的 建筑形体效果。这对于延续地域建筑特色风貌和传统文化 价值有着全新的意义。

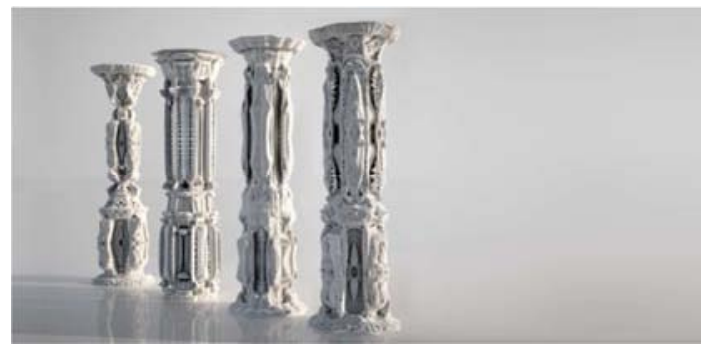

图8 根据不同外部参数细分得到的柱子。

英国剑桥大学教授John Frazer在其著作《一种进化 的建 筑》 (An Evolutionary Architecture. Architectural Association) 中将塔斯干柱式参数化, 以此为基础, 在不同的环境条件下, 演化出各种变体。迈 克尔 - 汉斯麦尔 (Michael Hansmeyer) 认为, 从美学的 角度, 任何形态都能以数学逻辑来看待, 并加以形式化和 编码化。他在 “第六柱式” (The 6th Order) 中通过计 算机迭代细分实现由外部参数 (地形和拓扑信息) 控制生
成形状各异的柱子, 把复杂的形态单元化和像素化, 通过 图层的切割和叠加来实现复杂的数字化形态（图8）。安 德鲁・桑德斯 (Andrew Saunders) 在AD杂志中介绍了伦 斯勒建筑学院（Rensselaer School of Architecture） 学生如何通过分析几何与数学逻辑, 使用参数化重新演绎 巴洛克式建筑（Re-Interpreting the Baroque）, 进行 巴洛克式建筑的设计（图9）。

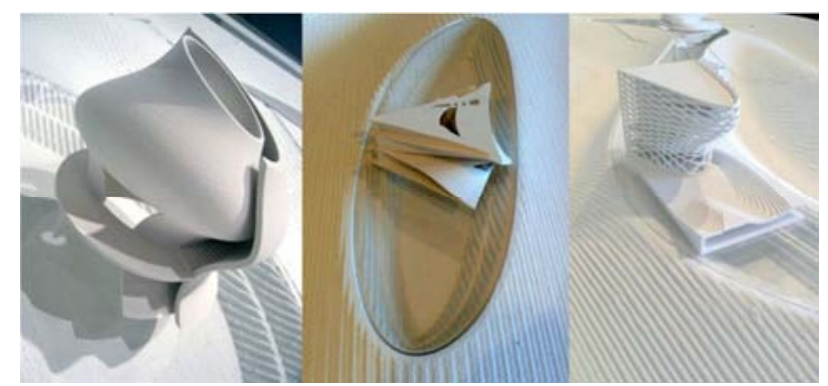

图9 巴洛克式的几何参数变化模型。

杨振宁在《美与物理学》中认为, 整个物理学最后的精 华是那为数不多、概括度极高且简洁优美的理论架构, 表现 为一些经典方程式。然而, 科学如诗如画般的审美意象, 是 非科学家很难观照、认知和体验到的, 即使物理学专业的人 也未必都能够全面深刻了解这些 “像诗一样的方程” 。[13] 就持久的艺术趣味而言, 科学公式所产生的审美信息是有限 的, 因而这种信息是一个封闭的系统。人们普遍因为不懂科 学的形式或者抽象的知觉实体, 所以对科学难以像伟大的科 学家们那样产生意和象, 融合感受科学意象, 进而产生科学 审美意象。同样, 在参数化设计中, 各种出人意料、千奇百 怪的结果的 “象” (图形、建筑形体等) 及其视觉经验必须依 据一个全新的审美标准来评判。格雷格・林恩 (Greg Lynn) 的胚胎学住宅 (Embryological House) 是使用玛雅软件设计 借助遗传算法生成的一系列住宅方案。（图10）其中的逻辑 关系是确定不变的, 每个房间严格按照一定的几何参数, 他 们都分为上下两层, 上层为卧室。当这些限定的 “意” 固定 下来后, 各种基于气候, 功能要求以及建筑材料等参数被带 入, 开始受精卵发育的分裂过程, 结果产生形状不同的设计 结果, 也就是不同形式的 “象”。

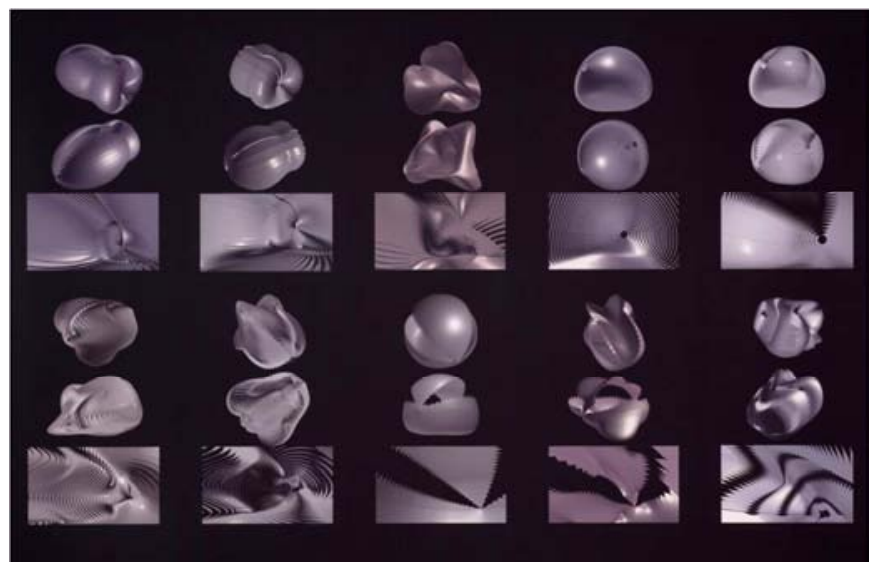

图10 格雷格 - 林恩的胚胎学住宅。 
参数化建筑的审美意象解读, 不是对单纯建筑形象做 传统的主观美学的评判, 其审美价值还隐含在建筑设计过 程中生成的那些逻辑关系之中。而这种潜在的、过程中的 程序算法的 “意” , 是比科学公式更难以接近人们通常感 知到的客观形式。因而对设计师隐含意图与作品深层逻辑 的揣摩对于建筑使用与欣赏者来说是一种徒劳, 于是使用 与欣赏者往往对建筑形象与象征意义之间产生多种解释 的可能, 参数化形成的复杂又新奇的形体带来的建筑审美 体验更具个体性与多义性, 解释的自由度增加。

\section{3. 参数化建筑审美过程的特殊性}

不同于功能决定形式的现代建筑设计, 参数化建筑设 计是经由程序逻辑生成建筑形式的过程。建筑师在参数化 设计中找到了形式主义以外的设计逻辑, 这些设计逻辑包 括了环境的、社会的、经济的或者文脉的等多方面的参数。 [14] 因此, 参数化设计的最重要的环节是, 借助参数化设 计软件, 将相关设计因素作为参数, 制定形态的逻辑生成 规则。建筑师不是直接控制形态的生成, 而是通过数学公 式或参数的改变控制生成逻辑的变化, 从而间接地控制形 态的生成。参数化建筑的概念构思过程常常被形容为 “用 代码写设计”。

参数化建筑审美离不开对相关参数化逻辑、程序与生 成规则的数字化理论的理解和掌握。对于相关建筑的生成 规则、理论与程序的观照, 是参数化建筑设计领域里特有 的审美体验, 审美主体如不具备相应参数化理论背景, 就 不具备相应的理解与欣赏能力, 进行参数化建筑审美评价 时便很难产生这一层次的美感。参数化建筑的美来源于对 建筑功能、形式、结构、环境行为心理等客观条件的理解 与分析, 它是这些客观因素在参数化思维中的凝结和升华, 也是设计者的参数化思维审美选择的结果。这种参数化建 筑审美更类似于对科学理论的审美, 只有在审美客体在审 美心理、审美意识以及理性思维达到一定阶段且相互渗透, 才能充分展现和发挥。因而, 参数化建筑这一层次的审美 需要相关参数化科学理论基础作支撑, 并不能被感官直接 感知。

由此可见, 参数化建筑的审美过程包含了科学理论与 艺术作品两种不同的审美观念, 有着审美形态特征上的两 个不同层次。即抽象性与形象性两个层次: 参数化编程过 程主要通过抽象方式，通过 “舍象取质” 将完整的表象抽 象为逻辑生成规则。而参数化建筑的形体生成过程中通过 “造象显质” 形象思维方式, 使最终生成的具象的参数化 建筑造型升华为感性的审美艺术形象。

\section{4. 结论}

数字化技术带来的建筑领域变革一日千里, 面对当前 迅猛发展的参数化建筑实践与纷繁复杂的建筑现象, 对参 数化建筑美学思潮的归纳与演绎, 有助于建立更加完善的 当代中国建筑美学理论体系, 有助于预测当前世界建筑美 学的发展趋势; 总结参数化建筑设计艺术表现手法, 能够
为拓展建筑创作视野、发展建筑创作理念, 提供新的艺术 理论基础和方法论指导; 从中国国情以及当代建筑师的角 度出发, 分析研究美学与参数化建筑艺术的问题, 能为参 数化建筑方案评价提供美学依据; 适应中国国情, 面向信 息社会的参数化建筑设计理论, 能为建筑人才的培养提供 理论借鉴。

\section{致谢}

本文为国家自然科学基金资助项目（No. 51408403） 及教育部人文社会科学研究规划基金（N0：13YJCZH006） 的阶段性成果之一。

\section{参考文献}

[1] 蔡良娃, 曾鹏. 参数化建筑设计中美学观念的演变 $[J]$. 新建 筑, 2013（2）:69。

[2] 蔡良娃, 曾鹏. 参数化建筑设计中美学观念的演变 $[J]$. 新建 筑, 2013（2）:71。

[3] 曾坚、蔡良娃, 建筑美学, 中国建筑工业出版社, 2010.36。

[4] 高岩、帕特里克・舒马赫. 晰释复杂性一一与扎哈 ・ 哈迪德 建筑师事务所合伙人帕特里克 - 舒马赫的访谈 $[J]$. 世界建 筑, 2006, (4): 10 。

[5] 张文化. 新媒体艺术: 建立在主体间性上的审美意义 [J]. 厦 门大学学报（社会科学版）. 2009, (2) : 122-128。

[6] Kas Oosterhuis. Swarm Architecture II[M]. Game Setand Match II. Episode Publisher, 2006。

[7］石孟良、彭建国、汤放华. 秩序的审美价值与当代建筑的美 学追求 $[J]$. 建筑学报. 2010, (4): 17 。

[8］［美]罗伯特・文丘里. 周卜臨译. 建筑的复杂性与矛盾性 [M]， 北京: 水利水电出版社, 2006. P32。

[9] http://www. generativedesign. com/asialink/de6. htm.

[10］ [瑞士 ]W ・ 博奥席耶。勒 ・柯布西耶全集第五 卷・1946-1952 [M]. 牛燕芳, 程超译. 北京: 中国建筑工 业出版社, 2005. 169。

[11] Kolarevic B. Architecture in the Digital Age Designand Manufacturing [M]. NewYork \& London : SponPress, 2003.

[12] 赵伶俐. 艺术意象·审美意象·科学意象一一创造活动心理 图像异同的理论与实证构想 $[\mathrm{J}]$. 自然辩证法研 究. 2007 (7) : 106。

[13] 李季宏. 科学意象初探 [J]. 自然辩证法研究. 1997 (2) : 19。

[14] 高隽璐. 当代参数化地域建筑设计中的符号学应用 $[\mathrm{J}]$. 建 筑与文化. 2016 (7) :221。 